\title{
Psoriasis: Vitamin-D-Mangel bei vier von fünf Patienten im Winter
}

\author{
Weitreichende Folgen werden einem Mangel an Vitamin D zugeschrieben - \\ von der Osteoporose über Autoimmunkrankheiten bis hin zu \\ kardiometabolischen Erkrankungen und Krebs. Thema einer italienischen \\ Studie waren Vitamin-D-Spiegel bei zwei Patientengruppen und Gesunden.
}

$\mathrm{n}$ einer Querschnittstudie wurden die Laborwerte von 145 Patienten mit chronischer Plaque-Psoriasis, 112 Patienten mit rheumatoider Arthritis und $141 \mathrm{Ge}$ sunden verglichen.

Die 25-Hydroxyvitamin-D-Werte (25$\mathrm{OH}-\mathrm{D})$ im Serum lagen bei den Psoriatikern (Frauen: 19,3 ng/ml, Männer: 21,2 $\mathrm{ng} / \mathrm{ml}$ ) signifikant niedriger als in der Kontrollgruppe mit 37,1 ng/ml. Zwischen den Patienten mit Psoriasis und rheumatoider Arthritis $(24,0 \mathrm{ng} / \mathrm{ml})$ zeigten die Durchschnittswerte keinen signifikanten Unterschied. Kalzium- und Parathormonwerte sowie die durchschnittliche Dauer der Sonnenexposition waren in allen drei Gruppen etwa gleich.
Während sich bei 57,8\% der Patienten mit Psoriasis ein Vitamin-D-Mangel von unter $20 \mathrm{ng} / \mathrm{ml}$ zeigte, lag dieser Anteil in der Gruppe mit rheumatoider Arthritis bei 37,5\% und in der Kontrollgruppe bei $29,7 \%$. Wie erwartet schwankten die Werte mit den Jahreszeiten. So stieg der Anteil der Patienten mit Vitamin-D-Mangel in allen drei Gruppen im Winter an - am deutlichsten bei den Psoriatikern mit einem Anteil von 80,8\% (rheumatoide Arthritis: 41,3\%, Kontrollgruppe: 30,3\%).

Fazit: Ein diagnostizierter Vitamin-DMangel kann behandelt werden. Allerdings, so geben die Autoren zu bedenken, existiert bislang kein Beweis, dass eine

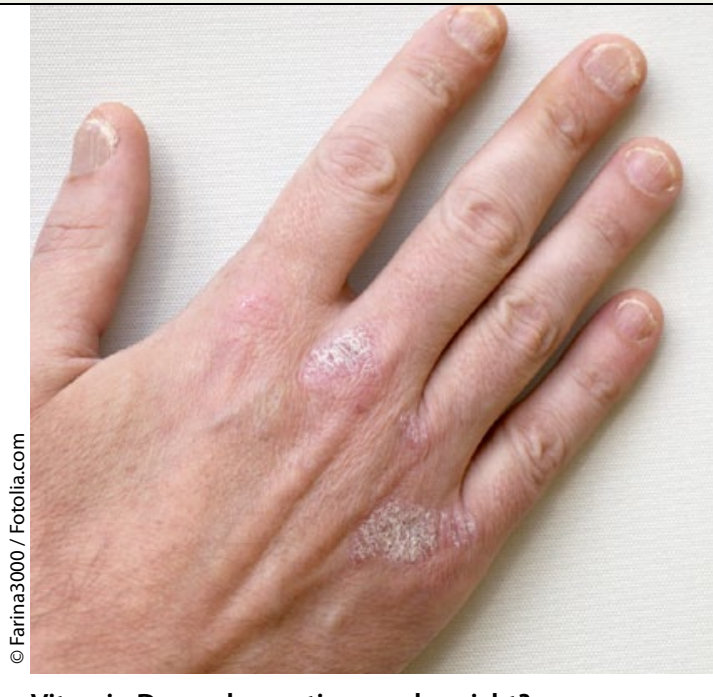

Vitamin D supplementieren oder nicht?

Vitamin-D-Supplementation das Entzündungsgeschehen verringert oder das Risiko für andere Autoimmunerkrankungen reduziert. Dennoch sollten Psoriasis-Patienten auf einen Vitamin-D-Mangel hin untersucht werden, um Maßnahmen zu ergreifen.

Dr. Christine Starostzik

Gisondi $P$ et al. Vitamin D status in patients with chronic plaque psoriasis. Br J Dermatol 2012; 166: 505-10

\section{Rückmutation beim Kindler-Syndrom}

\begin{abstract}
Einer internationalen Autorengruppe ist es gelungen, in Hautarealen von Patienten mit Kindler-Syndrom eine Rückmutation zu gesunden Keratinozyten zu beobachten - ein interessanter Ansatz für zukünftige Therapien?
\end{abstract}

$\mathrm{D}$ as Kindler-Syndrom ist durch Blasenbildung der Haut, Photosensitivität und Poikilodermie gekennzeichnet. Die autosomal-rezessive Störung geht auf Mutationen im FERMT1-Gen zurück. Das Gen kodiert für das Eiweiß Kindlin-1, ein intrazelluläres Linkerprotein für das Zytoskelett. Die Folge der Mutationen sind Spalten in der dermal-epidermalen Verbindung sowie Verdopplungen und Unterbrechungen der epidermalen Basalmembran. Interessanterweise zeigen sich bei den Patienten auf der Haut aber auch Inseln mit normalem Gewebe.

Die Forscher um Bruckner-Tuderman haben nun die Fälle von sechs Patienten beschrieben, die Duplikationsinsertionen in FERMT1 aufwiesen. Sie hatten ebenfalls das typisch bunte Hautmuster, das sich als genetisches Mosaik entpuppte: In bestimmten Hautbereichen wurde Kindlin-1 wieder normal exprimiert. Die Ursache hierfür war ein „Slipped Mispairing“. Dabei werden die Basen während der DNA-Replikation nicht korrekt gepaart. In den gesundeten Hautzellen der Patienten war gewissermaßen „vergessen“ worden, die fehlerhaften Insertionen nachzubauen - die Mutationen wurden dadurch rückgängig gemacht. An manchen Stellen hatte die mitotische Rekombination sogar für homozygot normale Keratinozyten gesorgt.

Die Reparatur von Kindlin-1 normalisierte das Gewebe klinisch und strukturell. Offenbar besitzen die gesunden Keratinozyten einen Selektionsvorteil, der es ihnen erlaubt, sich über Klonierung auszubreiten. Die Forscher hoffen nun, ihre Erkenntnisse für zelltherapeutische
Ansätze nutzen zu können, die sich spon$\tan$ geheilter Hautzellen bedienen. Profitieren könnten davon Patienten mit Kindler-Syndrom, aber auch Patienten mit weiteren Genodermatosen. Zumindest im Experiment ist es bei Epidermolysis bullosa bereits gelungen, rückmutierte Keratinozyten zu transplantieren. Für die breitere klinische Anwendung müsste es allerdings gelingen, die Anzucht der Zellen zu verbessern.

Fazit: Spontane Rückmutationen können den Hautbefund von Patienten mit Kindler-Syndrom partiell normalisieren. Es entsteht dabei ein genetisches Mosaik. Mit der Transplantation patienteneigener rückmutierter Zellen könnte die Option auf eine Therapie bestehen, Patienten mit Kindler-Syndrom und anderen Genodermatosen zu behandeln.

Dr. Robert Bublak

Kiritsi D et al. Revertant mosaicism in a human skin fragility disorder results from slipped mispairing and mitotic recombination. J Clin Invest 2012; 122: 1742-6 